\title{
Cloning and Expression of Mitochondrial Capsule Selenoprotein Gene in the Golden Hamster
}

\author{
Sang-Yoon NAM*, Seishi MAEDA, Masahiko FUJISAWA, Masamichi KUROHMARU and Yoshihiro HAYASHI \\ Department of Veterinary Anatomy, Graduate School of Agricultural and Life Sciences, The University of Tokyo, Bunkyo-ku, Tokyo 113- \\ 8657, Japan
}

(Received 30 April 1998/Accepted 19 June 1998)

\begin{abstract}
Mitochondrial capsule selenoprotein (MCS) has been known as a structural protein of the mitochondrial sheath in spermatozoa. In the present study, a full-length cDNA encoding the MCS was first isolated from the testes of 10-week-old golden hamsters using a RACE (rapid amplification of cDNA ends) technique and its mRNA expression pattern was investigated from the hamster tissues by reverse transcription-polymerase chain reaction (RT-PCR) analysis. Hamster MCS cDNA was 820 bp long, including 24 bp of the 5'untranslated region (UTR) and 243 bp of the 3 '-UTR, and showed identity of $75.6 \%$ and $73.9 \%$ with mouse and rat MCS. According to the deduced amino acid (aa) sequence analysis, hamster MCS encoded a polypeptide of 184 aa, including a cysteine- and proline-rich domain which is the characteristic sequences of MCS, and contained 2 in-frame UGA codons for selenocysteine. Hamster MCS also shared aa identity of $64.4 \%$ with mouse MCS and contained an Arg-Lys-Ser-Thr-rich region in the N-terminus similar to the mitochondrial targeting signal. On the other hand, according to the RT-PCR analysis using the specific primers for hamster MCS, hamster MCS mRNA was expressed in various tissues as well as the testes. This finding indicates that MCS in hamster may have more than just a function of mitochondrial sheath formation of spermatozoa._KEY WORDS: hamster, mitochondrial capsule selenoprotein, RACE, selenocysteine.
\end{abstract} J. Vet. Med.Sci. 60(10): 1113-1118, 1998

Selenium (Se) is an essential micronutrient for mammals [33] and its biological functions are mediated by a selenoprotein $[6,29,32]$. Se is incorporated into the selenoprotein by recognition of the stop codon UGA as a codon for selenocysteine [11]. Although labeling in vivo with ${ }^{75} \mathrm{Se}$ and subsequent SDS-PAGE with autoradiography has identified up to 30 such proteins, to date only 11 have been further characterized by purification and/ or cloning to determine their biochemical functions [4, 34]. It has been known that selenoproteins play the roles in the cell antioxidant system $[19,35]$, thyroid hormone metabolism [5], and maintenance of fertility [17].

Se has been known to play an important part in the male reproductive system. In studies of animals fed a Se-deficient diet, the Se retention was highest in the male reproductive organs. Abnormal spermatozoa with impaired motility due to characteristic midpiece damage were frequently observed in the animals [7, 36, 37]. Tracer studies using ${ }^{75} \mathrm{Se}$ demonstrated that Se was localized within the midpiece of spermatozoa [37] and that it was bound to a polypeptide of 15-20 kilodaltons $(\mathrm{kD})$ confined to the mitochondrial sheath $[8,9,20,26]$. This molecule has been shown in the bull and the rat to be a major component of the keratinous mitochondrial capsule $[10,27]$ and it has been characterized as a cysteine- and proline-rich selenoprotein $[9,26]$. Kleene et al. [17] reported that mitochondrial capsule selenoprotein (MCS) isolated from the mouse testis is a structural protein of the mitochondrial sheath of spermatozoa, which is a polypeptide rich in cysteine and proline residues and contains 3 in-frame UGA codons for selenocysteine in the 5 '-end [16]. On the other hand, recently, we demonstrated that the MCS mRNA level increases with aging, but decreases due to Se deficiency in the mouse testes [22, 23, 25]. These findings indicate that besides its involvement in the mitochondrial sheath formation of spermatozoa, MCS may have a novel role in the testes.

So far, the cDNA clones that encode MCS have been isolated from the testes of mouse [16, 17], rat [1], and human [2]. In this study, a full-length cDNA encoding MCS was first isolated from the hamster testes using a RACE (rapid amplification of cDNA ends) technique [13] and its mRNA expression pattern was investigated in the hamster tissues by reverse transcription-polymerase chain reaction (RTPCR) analysis.

\section{MATERIALS AND METHODS}

Animals: Male golden hamsters (Mesocricetus auratus) maintained as a closed colony in our laboratory under standardized laboratory conditions in an air-conditioned room (lights-on from 0500 to $1900 \mathrm{hr}$ ) with free access to feed (CLEA Ltd., Tokyo, Japan) and water were used. At 10 weeks of age, 10 male hamsters were sacrificed under pentobarbital anesthesia for the extraction of total RNAs.

Cloning of hamster MCS cDNA from testes: Total RNA was isolated from the testes of 10-week-old golden hamsters using an RNA extraction kit (Isogen; NPG, Toyama, Japan). RACE was performed as described in the 5'/3' RACE kit (Boehringer Mannheim, Tokyo, Japan) based on Frohman's protocol [13]. The first strand cDNA was synthesized using the total RNA $(5 \mu \mathrm{g})$ and an oligo d (T)-anchor primer 5'GACCACGCGTATCGATGTCGACTTTTTTTTTTTTTTTTV-3' (V=A, C or G) by AMV reverse transcriptase. To prepare a cDNA template of the hamster MCS for RACE, 
3'-RACE was performed using a primer 5'ATGGACTCACTAGACTG- CTG-3' (S; mouse MCS DNA exon I: nucleotides (nt) 28-47), designed from the sequence of mouse MCS DNA [16] and a PCR anchor primer 5'GACCACGCGTATCGAT-GTCGAC-3'. Then, the PCR product was directly subcloned into a plasmid pGEM-T Easy Vector (Promega, Tokyo, Japan) and sequenced using the fluorescence labeling method with a DNA sequencing kit and a laser fluorescent DNA sequencer (Pharmacia, Tokyo, Japan). The sequence result confirmed that the cDNA template is a partial fragment (797 bp) of hamster MCS cDNA. Thereafter, primers As1 (antisense 1; 5'GATGGGTCACTCA TCT TC-T T T-3'), As 2 (5'-TTTCTACTTTCCACTTCCAGTTGC-3'), and As3 (5'GCTATCTAAATGTCAGTATCACTA-3') for 5'-RACE were obtained from the cDNA fragment sequence. For the 5'-RACE, the first strand cDNA was prepared using the As3 primer. Prior to tailing reaction of the 3 '-end, the first strand cDNA was purified using a purification kit (High Pure PCR Purification kit; Boehringer Mannheim, Tokyo, Japan). To attach a known sequence to the 3'-end of the first strand cDNA, a homopolymeric tail (poly A+) was appended with terminal deoxynucleotidyl-transferase and dATP. PCR amplification was performed twice using the following primers: for the first PCR, the oligo $\mathrm{d}(\mathrm{T})$-anchor primer and the As2 primer; for the second PCR, the PCR anchor primer and the As1 primer. All PCR amplifications were carried out in a thermal cycler (Iwaki, Tokyo, Japan) using a protocol of an initial denaturing step at $95^{\circ} \mathrm{C}$ for 3 min; then 35 cycles at $95^{\circ} \mathrm{C}$ for $1 \mathrm{~min}$, at $60^{\circ} \mathrm{C}$ for $1 \mathrm{~min}$, and at $72^{\circ} \mathrm{C}$ for $1.5 \mathrm{~min}$; and a further extension at $72^{\circ} \mathrm{C}$ for $10 \mathrm{~min}$. Then, the PCR products of $5^{\prime}$-RACE were subcloned directly into the plasmid and sequenced using the fluorescence labeling method.

$R T-P C R$ analysis: To investigate the expression of hamster MCS mRNA in tissues, total RNAs were isolated from the various tissues of 10-week-old golden hamster. The first strand cDNA was synthesized using $5 \mu \mathrm{g}$ of each total RNA and an oligo d (T) primer (Gibco BRL, Tokyo, Japan) by the reverse transcriptase. One cDNA fragment for the hamster MCS was amplified by PCR using the S and As3 primers (760 bp). PCR amplification was carried out in the thermal cycler using a protocol of an initial denaturing step at $95^{\circ} \mathrm{C}$ for $3 \mathrm{~min}$; then 30 cycles at $95^{\circ} \mathrm{C}$ for $1 \mathrm{~min}$, at $60^{\circ} \mathrm{C}$ for $1 \mathrm{~min}$, and at $72^{\circ} \mathrm{C}$ for $1.5 \mathrm{~min}$; and a further extension at $72^{\circ} \mathrm{C}$ for $5 \mathrm{~min}$. For a positive control, the specific primers for hamster beta-actin (GenBank accession No. AF014363) were used in the PCR: 5'CGTGACATCAAAG- AGAAGCTGTGC-3' (hamster beta-actin cDNA; nt 685-708) and 5'GCTCAGGAGGAGCAATGATCTTGAT-3' (hamster beta-actin cDNA; nt 1060-1036). Then, the PCR product was directly subcloned into the same plasmid and was confirmed by sequencing as a cDNA clone of the hamster beta-actin. To ascertain the contamination of genomic DNA in the total RNA samples, the first cDNA synthesis was performed without the reverse transcriptase and it's product was used as a template for an RT-negative PCR. All PCR products were run on a $2 \%$ agarose gel in TBE buffer.

\section{RESULTS}

Characterization of hamster MCS cDNA: A cDNA fragment (797 bp) was isolated from the hamster testes by 3'-RACE using a primer derived from the exon I sequence of mouse MCS genomic DNA [16] and a PCR anchor primer and was sequenced. The sequence result confirmed that the cDNA is a partial fragment of the hamster MCS cDNA, exhibiting $75.5 \%$ identity to the corresponding sequence of the mouse MCS gene. To determine the full-length cDNA sequence of hamster MCS, 5'-RACE was performed. The full-length cDNA and deduced amino acid (aa) sequences are shown in Fig. 1. The hamster MCS cDNA was 820 bp long, including $24 \mathrm{bp}$ of the 5 '-untranslated region (UTR) and 243 bp of the 3 '-UTR, and contained 2 in-frame UGA codons for the potential selenocysteine at nt 43-45 and nt 124-126. The cDNA had 2 putative initiation codons for translation at nt 25-27 and nt 184-186. The initiation codon ATG at nt 25-27 and the termination codon TAG at nt 577579 predicts a translation product of 184 aa, containing the selenocysteine codon in the 5'-end [16]. The 3'-UTR possessed the polyadenylation signal AATAAA at nt 789794. The hamster MCS cDNA sequence showed identity of $75.6 \%$ and $73.9 \%$ with mouse [16] and rat [1] MCS (data not shown). As shown in Fig. 2, the deduced aa sequence of hamster MCS exhibited $64.4 \%$ identity to mouse MCS and was shorter than the sequence of mouse MCS, which was due to a deletion of aa at positions 81-94. A predicted molecular mass of the hamster MCS was about 20,099 D. The hamster MCS could be divided into 3 domains: an amino-terminal domain $(1-61 ; 61$ aa), a cysteine- and proline-rich domain (62-144; 83 aa), and a carboxy-terminal domain (145-184; 40 aa). The cysteine- and proline-rich domain contained about $61.5 \%$ of cysteine $(30.1 \%)$ and proline $(31.4 \%)$ residues, while the amino-terminal and the carboxy-terminal domains showed a low level of cysteine and proline residues $(11.5 \%$ and $5 \%)$. Moreover, the cysteine- and proline-rich domain consisted of about 7 repeats with a consensus sequence $\mathrm{X}$-Pro-Cys-Cys-Pro-X ( $\mathrm{X}$ is any aa) and 22 of the 25 cysteine residues occurred as dicysteine motifs in the domain. On the other hand, the MCS contained an Arg-Lys-Ser-Thr-rich region similar to a potential mitochondrial targeting signal [15] in the aminoterminal domain (small boxes): $\mathrm{Thr}^{35}$-Lys $^{36}$-Lys $^{37}-\mathrm{Ser}^{38}$ - Arg ${ }^{40}$ Lys $^{41}-\mathrm{Arg}^{46}-\mathrm{Ser}^{47}-\mathrm{Thr}^{49}$ - Thr $^{51}$ - Lys ${ }^{53}-$ Lys $^{54}$.

Expression of hamster MCS mRNA in various tissues: So far, MCS gene has been only detected from the testes of mouse [16], rat [1], and human [2]. To investigate the expression pattern of hamster MCS mRNA in tissues, total RNAs were isolated from testes, adrenal gland, brain, heart, hypophysis, kidney, liver, lung, skeletal muscle, pancreas, and submandibular gland of 10-week-old golden hamsters and were subjected to RT-PCR analysis using the S and 


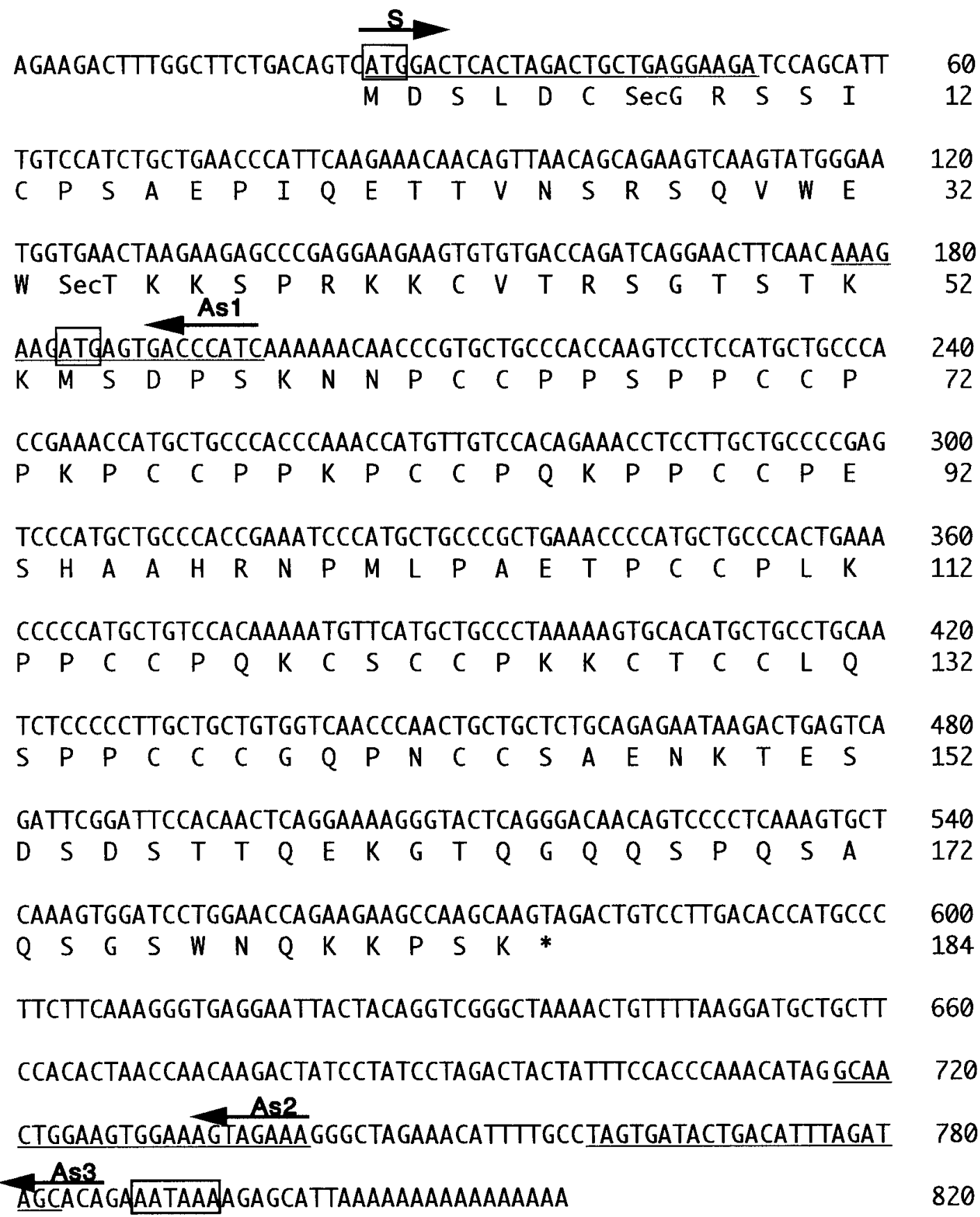

Fig. 1. Full-length cDNA and deduced aa sequences of hamster MCS. The underlined sequences (S, As1, As2, and As3; arrows) indicate the primers for the RACE protocol. 'Sec' represents the position of selenocysteine. The putative initiation codon ATG and polyadenylation signal AATAAA are boxed. The termination codon is observed at 577-579 (asterisk). The nucleotide sequence data will appear in the DDBJ, EMBL, and GenBank nucleotide sequence databases with the accession number AB007985.

As3 primers. As shown in Fig. 3, surprisingly, a transcript (760 bp) for the hamster MCS was detected in the testes, adrenal gland, brain, heart, hypophysis, liver, skeletal muscle, pancreas, and submandibular gland, while it was not expressed in the kidney and lung. In addition, a transcript (376 bp) of the hamster beta-actin as a positive control was detected in all tissues examined, whereas no signal was detected in the RT-negative PCR.

DISCUSSION

A full-length cDNA for hamster MCS was first isolated 
1 : MDSLDCXGRP SIYSICXNIQ ETTFNTENQV WKCXTKKSPR KNCVARSG巾 П KKMSDPSK Mouse $* * * * * * * * * \quad * * \quad * * * * * * \quad * * * * * * * * * * * * * * * * * * * * * * * * * * * *$ Consensus

1 : MDSLDCXGRS SICPSAEPIQ ETTVNSRSQV WEWXTKKSPR KKCVTRSGIS TI KKMSDPSK Hamster

$\uparrow$ (64.4\%)

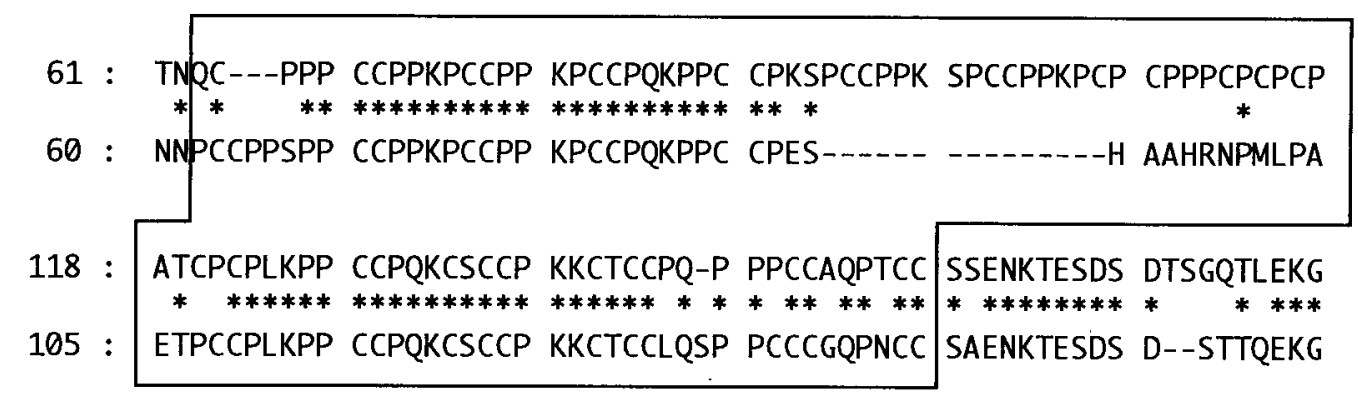

177 : SQSPQSPPGA Q-GNWNQKKS NK

163 : TQGQQSPQSA QSGSWNQKKP SK

Fig. 2. Comparison of the deduced aa sequence of hamster MCS to mouse MCS [16]. The numbers on the left side of sequences indicate the position of aa. The conserved aa residues are asterisked (consensus). X indicates the position of selenocysteine (arrows). The large boxed sequences represent a cysteine- and proline-rich domain, which is the characteristic sequences of MCS. The small boxed sequences indicate aa residues similar to a potential mitochondrial targeting signal [15]. The dashed lines (-) represent a deletion region of aa at positions 81-94 relative to that of mouse MCS.

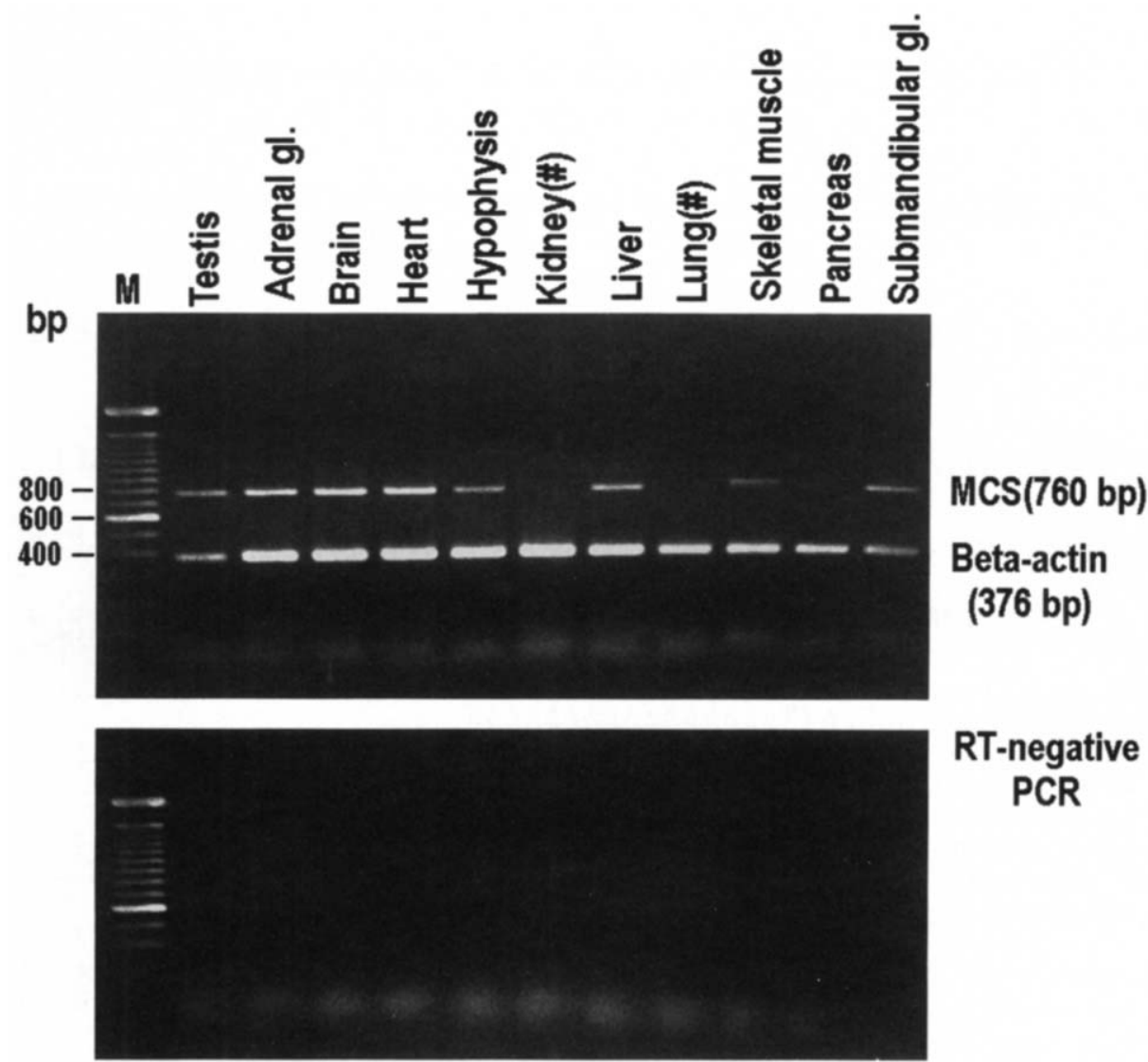

Fig. 3. Expression pattern of hamster MCS mRNA in various tissues. The upper panel shows a specific cDNA transcript (760 bp) of hamster MCS and a cDNA fragment of hamster beta-actin (376 bp) as a positive control. The lower panel represents the result of RT-negative PCR as a negative control. Except for the kidney and the lung, MCS signal is detected in all tissues examined. 
from the testes using a RACE technique [13]. The cDNA sequence shares identity of $75.6 \%$ and $73.9 \%$ with mouse MCS [16] and rat MCS [1], while it shows low identity of $24.1 \%$ with human MCS [2] (data not shown). The nucleotide sequence analysis of the hamster and mouse MCS cDNAs reveals that 5'- and 3'-UTRs are more conserved $(87.5 \%$ and $79.3 \%)$ than the coding region $(73.7 \%)$. These findings may be an indication of the importance of these sequences in the control of translation initiation and regulation of mRNA stability.

MCS has been characterized as a cysteine- and prolinerich protein of the testis $[1,2,9,17,26]$. The deduced aa sequence of mouse [16] shows $79 \%$ identity with the corresponding aa sequence of rat [1], whereas it reveals low identity (39\%) with that of human [2]. In the present study, hamster MCS contains a cysteine- and proline-rich domain $(30.1 \%$ and $31.4 \%)$ and shows aa identity of $64.4 \%$ with the mouse MCS and identity of $57.2 \%$ with the corresponding aa sequence of rat MCS. These findings indicate that this full-length cDNA may encode the MCS specific for hamster.

MCS appears as a protein of 20-21 kD in the testes of bull [26] and mouse [16], while it is expressed as a polypeptide of $15-17 \mathrm{kD}$ in rat testes $[1,9,10]$. Karimpour et al. [16] showed that mouse MCS encodes a polypeptide of 197 aa and contains 3 in-frame UGA codons for selenocysteine in the 5'-end. However, Adham et al. [1] reported that rat MCS encodes a polypeptide of 145 aa similar to a truncated clone of mouse MCS [17] and lacks the UGA codon for selenocysteine. In the present study, hamster MCS encodes a polypeptide of $184 \mathrm{aa}$, shorter than mouse MCS, which is due to a deletion of aa at positions 81-94, and contains 2 in-frame UGA codons in the same position of mouse MCS. Also, the predicted molecular mass of hamster MCS was about $20 \mathrm{kD}$. These findings suggest that MCS may be a selenoprotein of about $20 \mathrm{kD}$ in the testes.

On the other hand, phospholipid hydroperoxide glutathione peroxidase (PHGPx), an antioxidant selenoenzyme of 197 aa, is highly expressed in testes [18, $21,24,30]$. The PHGPx gene has shown to be transcribed as 2 forms in tissues: a mitochondrial form having a mitochondrial targeting signal in the N-terminus (197 aa; $23 \mathrm{kD})$ and a nonmitochondrial form (170 aa; $20 \mathrm{kD})$ [3, 22, 28]. In testes, PHGPX is mainly localized in the mitochondrial membrane as the mitochondrial form $[14,22$, 31]. As shown in Fig. 2, the hamster and mouse MCSs contains an Arg-Lys-Ser-Thr-rich region in the aminoterminal domain similar to the mitochondrial targeting sequences of mitochondrial membrane proteins [15]. This provides a possibility that MCS as a structural protein of mitochondrial sheath of spermatozoa, may have a mitochondrial targeting signal in N-terminus.

In this RT-PCR analysis using the specific primers for the hamster MCS, a transcript of about 760 bp was detected in many tissues as well as the testes. As shown in Fig. 1, the PCR product contained 2 in-frame UGA codons for selenocysteine in the 5'-end. These findings suggest that
MCS may act as a selenoprotein containing $\mathrm{Se}$ in the hamster tissues. To date, MCS gene has been known to be expressed only in the testes of bull [26], mouse [17], rat [1], and human [2] as a structural protein of mitochondrial sheath of spermatozoa [1, 2, 17]. However, as shown in Fig. 3, the MCS mRNA was expressed in various tissues of hamster. It is unknown why MCS gene in the hamster is expressed in many tissues differently from the above animals. On the other hand, we recently demonstrated that MCS mRNA in the mouse testis increases with aging, but remarkably decreases due to Se deficiency [23, 25]. These findings indicate that MCS may have more than just a function of mitochondrial sheath formation in spermatozoa.

A possible role of MCS in testes may be an antioxidant system to protect genetic materials (e.g., mitochondrial DNA) from harmful reactive oxidants in the spermatogenic cells undergoing rapid proliferation and differentiation during spermatogenesis as in other selenoproteins [19, 35].

ACKNOWLEDGMENT. This study was supported in part by Grant-in-Aid for Scientific Research Fund from the Ministry of Education, Science, Sports and Culture, Japan.

\section{REFERENCES}

1. Adham, I.D., Tessmann, D., Soliman, K.A., Murphy, D., Kremling, H., Szpirer, C. and Engel, W. 1996. Cloning, expression, and Chromosomal localization of the rat mitochondrial capsule selenoprotein (MCS): The reading frame does not contain potential UGA selenocysteine codons. DNA Cell Biol. 15: 159-166.

2. Aho, M., Schwemmer, D., Tessmann, D., Murphy, D., Mattei, G., Engel, W. and Adham, I.M. 1996. Isolation, expression, and chromosomal localization of the human mitochondrial capsule selenoprotein gene (MCSP). Genomics 32: 184-190.

3. Arai, M., Imai, H., Sumi, D., Imanaka, T., Takano, T., Chiba, N. and Nakagawa, Y. 1996. Import into mitochondria of phospholipid hydroperoxide glutathione peroxidase requires a leader sequence. Biochem. Biophys. Res. Commun. 227: 433439.

4. Arthur, J.R. and Beckett, G.J. 1994. New metabolic roles for selenium. Proc. Nutr. Soc. 53: 615-624.

5. Arthur, J.R., Nicol, F. and Beckett, G.J. 1993. Selenium deficiency, thyroid hormone metabolism, and thyroid hormone deiodinases. Am. J. Clin. Nutr. (Suppl.) 57: 236S-239S.

6. Berry, M.J., Banu, L. and Larsen, P.R. 1991. Type I iodothyronine deiodinase is a selenocysteine-containing enzyme. Nature(Lond.) 349: 438-440.

7. Brown, D.G. and Burk, R.F. 1973. Selenium retention in tissues and sperm of rats fed a Torula yeast diet. J. Nutr. 102: 102-108.

8. Calvin, H.I. 1978. Selective incorporation of selenium-75 into a polypeptide of the rat sperm tail (1). J. Exp. Zool. 204: 445-452.

9. Calvin, H.I., Cooper, G.W. and Wallace, E. 1981. Evidence that selenium in rat sperm is associated with a cysteine-rich structural protein of the mitochondrial capsules. Gamete Res. 4: 139-149.

10. Calvin, H.I., Grosshans, K., Musicant-Shikora, S.R. and Turner, S.I. 1987. A developmental study of rat sperm and 
testis selenoproteins. J. Reprod. Fertil. 81: 1-11.

11. Chambers, I., Frampton, J., Goldfarb, P., Affara, N., McBain, W. and Harrison, P.R. 1986. The structure of the mouse glutathione peroxidase gene: the selenocysteine in the active site is encoded by the 'termination' codon, TGA. EMBO J. 5: 1221-1227.

12. Chomczynski, P. 1992. One-hour downward alkaline capillary transfer for blotting of DNA and RNA. Anal. Biochem. 201: 134-139.

13. Frohman, M.A., Dush, M.K. and Martin, G.R. 1988. Rapid production of full-length cDNAs from rare transcripts: Amplification using a single gene-specific oligonucleotide primer. Proc. Natl. Acad. Sci. U.S.A. 85: 8998-9002.

14. Godeas, C., Sandri, G. and Panfili, E. 1994. Distribution of phospholipid hydroperoxide glutathione peroxidase (PHGPx) in rat testis mitochondria. Biochim. Biophys. Acta 1191: 147150.

15. Hurt, E.C. and van Loon, A.P.G.M. 1986. How proteins find mitochondria and intramitochondrial compartments. Trends Biochem. Sci. 11: 204-206.

16. Karimpour, I., Cutler, M., Shih, D., Smith, J. and Kleene, K.C. 1992. Sequence of the gene encoding the mitochondrial capsule selenoprotein of mouse sperm: Identification of three in-phase TGA selenocysteine codons. DNA Cell Biol. 11: 693-699.

17. Kleene, K.C., Smith, J., Bozorgzadeh, A., Harris, M., Hahn, L., Karimpour, I. and Gerstel, J. 1990. Sequence and developmental expression of the mRNA encoding the seleno-protein of the sperm mitochondrial capsule in the mouse. Dev. Biol. 137: 395-402.

18. Lei, X.G., Evenson, J.K., Thompson, K.M. and Sunde, R.A. 1995. Glutathione peroxidase and phospholipid hydroperoxide glutathione peroxidase are differentially regulated in rats by dietary selenium. $J$. Nutr. 125 : 1438-1446.

19. Little, C., Olinescu, R., Reid, K.G. and O'Brien, P.J. 1970. Properties and regulation of glutathione peroxidase. J. Biol. Chem. 245: 3632-3636.

20. McConnell, K.P., Burton, R.M., Kute, T. and Higgins, P.J. 1979. Selenoproteins from rat testis cytosol. Biochim. Biophys. Acta 588: 113-119.

21. Nam, S.-Y., Fujisawa, M., Kim, J.-S., Kurohmaru, M. and Hayashi, Y. 1998. Expression pattern of phospholipid hydroperoxide glutathione peroxidase messenger ribonucleic acid in mouse testis. Biol. Reprod. 58: 1272-1276.

22. Nam, S.-Y., Kurohmaru, M. and Hayashi, Y. 1998. Testicular selenoproteins: Expression and distribution. pp. 239-245. In: Reproductive Biology Update: Novel Tools for Assessment of Environmental Toxicity (Miyamoto, H. and Manabe, N. eds.), Nakanishi Printing Co., Kyoto.

23. Nam, S.-Y., Maeda, S., Ogawa, K., Kurohmaru, M. and Hayashi, Y. 1997. Expression pattern of the mitochondrial capsule selenoprotein mRNA in the mouse testis after puberty; in situ hybridization study. J. Vet. Med. Sci. 59: 983-988.

24. Nam, S.-Y., Nakamuta, N., Kurohmaru, M. and Hayashi, Y.
1997. Cloning and sequencing of the mouse cDNA encoding a phospholipid hydroperoxide glutathione peroxidase. Gene 198: 245-249.

25. Nam, S.-Y., Youn, H.-Y., Ogawa, K., Kurohmaru, M. and Hayashi, Y. 1997. Expression of mitochondrial capsule selenoprotein mRNA increases with aging, but decreases by selenium deficiency in the mouse testis. J. Reprod. Dev. 43: 227-234.

26. Pallini, V. and Bacci, E. 1979. Bull sperm selenium is bound to a structural protein of mitochondria. J. Submicr. Cytol. 11: 165-170.

27. Pallini, V., Baccetti, B. and Burrini, A.G. 1979. A peculiar cysteine-rich polypeptide related to some unusual properties of mammalian sperm mitochondria. pp. 141-151. In: The Spermatozoon (Fawcett, D.W. and Bedford, J.M. eds.), Urban \& Schwarzenberg, Baltimore-Munich.

28. Pushpa-Rekha, T.R., Burdsall, A.L., Oleksa, L.M., Chisolm, G.M. and Driscoll, D.M. 1995. Rat phospholipid-hydroperoxide glutathione peroxidase; cDNA cloning and identification of multiple transcription and translation start sites. J. Biol. Chem. 270: 26993-26999.

29. Rotruck, J.T., Pope, A.L., Ganther, H.E., Swanson, A.B., Hafeman, D.G. and Hoekstra, W.G. 1973. Selenium: Biochemical role as a component of glutathione peroxidase. Science 179: 588-590.

30. Roveri, A., Maiorino, M. and Ursini, F. 1994. Enzymatic and immunological measurements of soluble and membrane-bound phospholipid-hydroperoxide glutathione peroxidase. Methods Enzymol. 233: 202-212.

31. Roveri, A., Maiorino, M., Nisii, C. and Ursini, F. 1994. Purification and characterization of phospholipid hydroperoxide glutathione peroxidase from rat testis mitochondrial membranes. Biochim. Biophys. Acta 1208: 211-221.

32. Schuckelt, R., Brigelius-Flohe, R., Maiorino, M., Roveri, A., Reumkens, J., Straßburger, W., Ursini, F., Wolf, B. and Flohe, L. 1991. Phospholipid hydroperoxide glutathione peroxidase is a selenoenzyme distinct from the classical glutathione peroxidase as evident from cDNA and amino acid sequencing. Free Rad. Res. Comms. 14: 343-361.

33. Schwarz, K. and Foltz, C.M. 1957. Selenium as an integral part of factor 3 against dietary necrotic liver degeneration. $J$. Am. Chem. Soc. 39: 3292-3293.

34. Sunde, R.A. 1994. Intracellular glutathione peroxidases-Structure regulation and function. pp. 45-77. In: Selenium in Biology and Human Health (Burk, R.F. ed.), Springer-Verlag, New York.

35. Ursini, F., Maiorino, M. and Gregolin, C. 1985. The selenoenzyme phospholipid hydroperoxide glutathione peroxidase. Biochim. Biophys. Acta 839: 62-70.

36. Watanabe, T. and Endo, A. 1991. Effects of selenium deficiency on sperm morphology and spermatocyte chromosomes in mice. Mutation Res. 262: 93-99.

37. Wu, A.S.H., Oldfield, J.E., Shull, L.R. and Cheeke, P.R. 1979. Specific effect of selenium deficiency on rat sperm. Biol. Reprod. 20: 793-798. 